\title{
The Virtual Shoe Salon: A creative and active approach for teaching research and data analysis to fashion students
}

\section{Naomi Joanna Braithwaite}

School of Art and Design, Nottingham Trent University, United Kingdom.

\begin{abstract}
The push towards creative and active learning in higher education has gained momentum in recent years, creating opportunities for innovative, student focused approaches. The Covid-19 pandemic shifted the educational landscape to the online world, propelling the need to create compelling learning activities for students. While also fostering a sense of community and facilitating both peer relationships and peer learning. This paper presents the development and initial implementation of an innovative teaching tool, The Virtual Shoe Salon, which is based on creative pedagogies and experiential learning. The Virtual Shoe Salon has been implemented to embed stronger skills in research and data analysis within the curriculum of our fashion business courses. As a teaching method it can embrace both online and face to face environments. Drawing from a theoretical foundation of material culture, combined with an adaptation of the photovoice research approach, the salon takes the ordinary, but expressive objects of shoes to engage students in research and analysis through collaboration, conversation and peer to peer learning. The paper presents the rationale for the Virtual Shoe Salon, and findings from its implementation with 250 students. Subsequent evaluation and reflection with both students and staff have evidenced the positive role of the Virtual Shoe Salon in facilitating active and collaborative learning around research and data analysis. While it has actively encouraged a dynamic and collaborative learning environment, the Virtual Shoe Salon has initiated a shared space underpinned by the sense of community and belonging for students that Covid-19 had eroded.
\end{abstract}

Keywords: Research skills; data analysis; shoes; images; material culture; collaborative learning. 


\section{Introduction}

The shoe, often considered to be the most ubiquitous of all clothing items, is well theorised for expressing and mediating meaning for its wearer (Brydon, 1998). Existing research from the fields of anthropology (Hockey, Dilley, Robinson \& Sherlock, 2014) and sociology (Belk, 2003) acknowledges shoes as expressions of self. The nature of shoes as ordinary, mundane, yet expressive objects, presented an intriguing case for their potential as a teaching tool for undergraduate and postgraduate students, undertaking fashion business courses. So came the idea for the Virtual Shoe Salon, a method for developing skills in research and data analysis that could be used within our Fashion Management, Marketing and Communication courses, with a view to evaluate its wider potential across disciplines. This paper presents the case for the Virtual Shoe Salon and discusses its development, implementation and evaluation as a creative and relatable tool for teaching approaches within the fields of qualitative research methods and data analysis. While its initial purpose was to bring a more interactive and experiential approach to teaching these subjects, it has also created a space for students to share stories on identity, generating cultural awareness within the discipline and fostering a sense of community and belonging, which the Covid-19 pandemic had impacted.

\section{Educational context}

The Virtual Shoe Salon responds to an institutional call to develop more inclusive and personalised approaches within the curriculum, that could translate across the physical and digital educational landscape. Ongoing curriculum reviews have evidenced the need for our fashion business students to build stronger skills in qualitative research and the analysis of real time data. Skills that will support the development of academic rigor but that are also integral to their future employment. The World Economic Forum (2018) predicted that an increase in data driven skills will be required in the changing landscape, and as educators this presents an opportunity to reappraise how we can embed those skills within our curriculum.

The Covid-19 pandemic forced higher education institutions to suspend face to face teaching in the Spring of 2020 and to create online learning environments that could facilitate student engagement and inclusive learning (Wahab, 2020), while encouraging a sense of belonging (Read, Archer \& Leathwood, 2003). While this sudden change to the academic year brought unprecedented challenges, with little time to plan for a fully online curriculum (Mishra, Gupta \& Shree, 2020), it also presented opportunities for creating innovative, student led approaches to learning, and it is this which the Virtual Shoe Salon responds to. 


\section{The Virtual Shoe Salon}

As an ethnographic researcher who uses photography, interviews and narrative storytelling around shoes and clothing in her own work, the author has noted the benefits to students of applying a research led approach to teaching qualitative research methods and approaches for data analysis. Course and module level student evaluations have highlighted the value and enjoyment that many students say they have gained from learning about process through real world research data. However, these previous approaches have focused more on the listening through lectures model, followed by seminar discussions, rather than encouraging students to create their own data through which they can interact collaboratively with the process and outcome. The pedagogical approach that underpins The Virtual Shoe Salon is the continued recognition of the value of creativity in fostering learning within higher education (Gustina \& Sweet, 2014). Creative pedagogies that are experientially defined are known to stimulate student engagement, through discovery and the co-creation of knowledge (Deniston-Trochta, 2003). As successful learning is facilitated through the activation of different senses (Biggs \& Tang, 2007); applying an experiential and interactive approach to the teaching of research and data analysis should facilitate and encourage student participation through problem-solving and the co-creation of knowledge.

The Virtual Shoe Salon is realised through a workshop activity focused around student led research, collaborative discussions and the co-creation of knowledge through the analysis of data. In advance of the workshop students are given a pre-session task, which asks them to engage in visual research by taking a shoe selfie. In addition, the students are required to provide a written response to the following two questions about their shoes; what do these shoes mean to you and how do you feel when you wear them

The shoe images and supporting responses to the questions are then posted in The Virtual Shoe Salon on Padlet (figure 1). Students will share these in small group discussions through a task titled 'interview with my shoes'. This process enables the students to develop skills in visual and qualitative research and to share knowledge of lived experience and feelings around identity. As an example of active and experiential learning this method engages students in research and will generate data which can be stored anonymously (with consent) in a student specific database. 


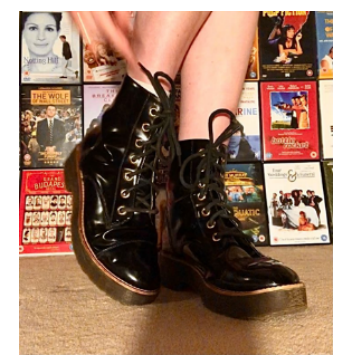

\section{My 'Everyday Battle' boots}

What do these shoes mean to me?

The pair of shoes that bonded me and my flatmate, (now close friend) Millie, on our first scary, awkward charity shopping quest in Nottingham. Having only known her a day, she found me these... in my size... for $£ 4$. It's fair to say that these unmatched shopping skills, have made us partners in crime, and not helped our shoe hoarding obsession. I've worn them through every uni adventure soo far! They've met new people with me, explored a new home, seen late nights and early mornings, danced with me, witnessed me laugh and cry and caused me to fall over a lot! So... they mean a great deal to me, and make the blisters worth it, for the memories that they hold... and Millie can borrow them any time :)

How do I feel when I wear them?

They just make me feel cool. They're battered and scraped and second hand, and yet they make me feel like a kick ass woman! They fit my androgynous style and the heavy soles mean you can hear me coming, but I love I stomping around Nottingham in them. The extra inches boost my confidence and have caused some height arguments with my male friends, which are always a laugh. I just love them. They're not branded, designer or very fashionable, but they're reliable and that's all I need. Once lockdown is over, I'Il be wearing them out until they wear out!!! :D

Figure 1. Padlet Post-Battle Boots.

The workshop then comprises an interactive peer focused session which due to the continued Covid restrictions is delivered through Microsoft Teams. Using Teams' break-out rooms, students work in small groups and follow the set of tasks detailed below.

1. Interview with my shoes: having learned about visual and qualitative research through lectures the students are tasked to enter the Virtual Shoe Salon on Padlet and share their images and narrative responses with each other. This approach draws from the methodology of photo elicitation which uses photographs as a stimulus to generate discussions which interpret meaning and knowledge (Glaw, Inder, Kable \& Hazelton, 2017). Photo elicitation is an invaluable process for collaborative discussions that explore the deeper multi layers of meanings that images, along with the written narratives, can stimulate (Harper, 2002). As an approach it enables the generation of different types of knowledge as it can evoke individual emotions and memories (Harper, 2002), thus encouraging a personalised learning experience.

Following initial conversations, the students will assume the role of an interviewer and develop a line of questioning that will enable them to elicit deeper understanding about the meaningfulness of shoes for each individual within their 
group. Sessions with undergraduate students have an interview template guide to follow, but postgraduates are encouraged to work independently, developing questions organically. Coming together as a larger group we reflect and share their initial thoughts about their process of research and questioning. They are asked to debate the value and challenges that this type of research process can elicit, with the objectives of understanding its application to their studies, as well as its role in understanding consumers in the fashion industry.

2. Collaborative Conversations: returning to their small groups the students work together to analyse their data and interpret meaning from the images, written narratives and interviews that they have undertaken. Through collaboration they can share meanings, stories and experiences and thus generate a dataset of images and narratives, that can become a longitudinal, educational resource of images and text, created by students. The intention with this approach is to encourage skills in creative thinking, collaboration, problem solving and co-creation of knowledge with a focus on understanding individual identity and personal values. This method privileges the sharing of students' own knowledge and experiences and supports a personalised learning strategy, supported by the Institution.

The learning objectives that define the Virtual Shoe Salon are listed below:

- An interactive process for learning about research and data analysis

- The creation of a longitudinal student specific dataset of images and text

- An active and experiential tool for learning skills in data generation, management and analysis

\section{Methodological and Theoretical Foundation}

Schell, Ferguson, Hamoline, Shea and Thomas-MacLean's (2009) work on photovoice as a teaching tool inspired the design of the Virtual Shoe Salon as a teaching method. In higher education photovoice has been used by students to capture their emotions and thoughts about a phenomenon through the lens of the photographs that they have taken (Wass, Anderson, Rabello, Golding, Rangi \& Eteuati, 2019). Like the traditions of photovoice our students have taken photographs of their shoes through which they can share their emotions and experiences of everyday life. Through this process they are engaging in qualitative and visual research and generating data that can be interpreted collaboratively through peer discussions.

The theoretical foundation of the Virtual Shoe Salon is grounded in material culture. Drawing from the work of Belk (2003) shoes are theorised as extensions of self and repositories of memory and thus symbolic of personal identity (McCarroll, 2019). Within the parameters of material culture (Woodward, 2019) shoes are relational objects through which individual and 
social experience are constructed. In this context they become valuable tools for developing a teaching resource that is relatable to students. The ordinariness of shoes means that even when an individual seemingly does not have an obvious meaning attached to them, they still, through their mundaneness, have some communicative powers, which can be drawn out through the research and analysis. Wearing clothing is one of the most ubiquitous aspects of human behaviour. Psychology recognises the diverse and powerful dynamic that clothing has in influencing the internal and external thought processes and moods of individuals (Adam $\&$ Galinsky, 2012). Given that the essence of qualitative research is about understanding the why of a phenomenon, and that meaning is derived from human interaction with the world, the ubiquitous nature of the shoe makes it an ideal medium for engaging research.

\section{Evaluation}

During the 2020/21 academic year the Virtual Shoe Salon has been implemented with 171 undergraduate students and 86 postgraduate students across our Fashion Management, Marketing and Communication courses. This has generated over 250 images and narratives which have been analysed to create an additional thematic dataset of meanings that have been interpreted through the students' collaborative conversations. Reflections with students about the virtual salon method have been undertaken during the sessions using a series of questions to which they have responded through a digital template. This forms part of their learning process, but also is a means of evaluating the success and wider impact of the Virtual Shoe Salon. Consensus has been that the students enjoyed participating in the Virtual Shoe Salon and in addition to feeling they had gained skills in research and analysis it enabled them to apply a personal approach to learning. Some of the comments are detailed below:

"It was fun and really interesting to learn about what things mattered to each of us". (participant A1)

"It is nice to do something practical and to apply what we are learning”. (participant F2)

"It has opened eyes to what you can you learn about research process and meaning through something so ordinary as a shoe". (Participant C7)

While they fully immersed themselves in the pre-session research activity, the workshop sessions stimulated impassioned conversations and debates around meaning between each other. When often silence has pervaded the digital classroom in group discussions, the Virtual Shoe Room seemed to generate chatter, debate and reflection. While the tool's role in raising awareness of identity around self and others was not a key factor in its development, it is an aspect that has come through strongly in the sessions and became integral to fostering the development of skills in data analysis. The images and narratives that the students have produced have been integral to encourage sharing and collaborative learning and this 
personalised approach has raised insights around cultural awareness. A conversation around a pair of DM boots highlighted why a student had made this purchase on arrival in the UK from her home country of Indonesia, where wearing such a rebellious style would be frowned upon. Through their shoe images the students were able to share their own experiences and views on identity within a cultural context.

In an increasingly digital world, where much of the academic year has been spent away from campus, an activity which focuses on the individuals and their interests became an important method for encouraging collaboration and developing a stronger sense of community between the year groups.

"We have learned so much about each other. It has been really interesting just to chat and share the experiences and through that learn how meaning can be drawn from an ordinary, everyday object" (participant E2).

The impact that the Virtual Shoe Salon has on tutor experience is of significance. Post session evaluations found that it engaged students positively in learning about research and data analysis through practice, rather than the usual rather passive format of taught sessions

\section{Concluding Remarks}

Initial trials with the Virtual Salon have demonstrated that it has value in encouraging students to learn about research and analysis through active participation. By facilitating collaborative conversations, the students can work together to make sense of data through the sharing of values, feelings and experiences. The ubiquitous nature of the shoe has opened dialogues around ideals, identity and cultural understanding, enabling them to learn about what things matter to each other and why. Through this process they have engaged with the process of data analysis with the opportunity to reflect on the deeper meanings of their shoes. In its next phase of development, the Virtual Shoe Salon will be trialed with students from English, Creative Writing, Journalism and Psychology to evaluate its potential across an interdisciplinary landscape. As an innovative approach, the Virtual Shoe Salon places students at the centre of learning and creates a visual and textual longitudinal resource which can continue to educate and inspire students beyond the discipline.

\section{References}

Adam, H. and Galinsky, A. (2012). Enclothed Cognition. Journal of Experimental Social Psychology, 48, 4, 918-925. https://doi.org/10.1016/j.jesp.2012.02.008

Belk, R. (2003). Shoes and Self. Advances in Consumer Research, 30, 27-33. 
Biggs, J., \& Tang, C. (2007). Using Constructive Alignment in Outcomes-Based Teaching and Learning Teaching for Quality Learning at University (3rd ed.) Maidenhead: Open University Press.

Brydon, A. (1998). Sensible Shoes. In A. Brydon, \& S. Niessen (Eds.), Consuming Fashion: Adorning the Transnational Body (pp.1-22). Oxford: Berg Publishers.

Deniston-Trochta, G. (2003). The Meaning of Storytelling as Pedagogy. Visual Arts Research 29, 57, 103-108.

Glaw, X., Inder, K., Kable, A., \& Hazelton, M. (2017). Visual Methodologies in Qualitative Research. Autophotography and Photo Elicitation Applied to Mental Health Research. International Journal of Qualitative Methods, 16, 1. https://doi.org/10.1177/1609406917748215

Gustina, C., \& Sweet, R. (2014). Creatives teaching creativity. The International Journal of Art and Design Education, 33, 1, 46-54. https://doi.org/10.1111/j.14768070.2014.01778.x

Harper, D. (2002). Talking about pictures: A case for photo elicitation. Visual Studies, 17, $13-26$.

Hockey, J., Dilley, R., Robinson, V., \& Sherlock, A. (2014). The Temporal Landscape of Shoes: A Life Course Perspective. The Sociological Review, 62, 255-275. https://doi.org/10.1111\%2F1467-954X.12154

McCarroll, C. (2018). Looking at the Self: Perspectival Memory and Personal Identity. $\begin{array}{llll}\text { Philosophical } & \text { Explorations, } & 22, & 3,\end{array}$ https://doi.org/10.1080/13869795.2018.1562087

Mishra, L., Gupta, T., \& Shree, A. (2020), Online teaching-learning in higher education during lockdown period of COVID-19 pandemic. International Journal of Educational Research Open, 1. https://doi.org/10.1016/j.ijedro.2020.100012.

Read, B., Archer, L., \& Leathwood, C. (2003). Challenging cultures? Student conceptions of 'belonging' and 'isolation' at a post-1992 university. Studies in Higher Education 28, 3, 261-277. https://doi.org/10.1080/03075070309290

Schell, K., Ferguson, A., Hamoline, R., Shea, J., \& Thomas-MacLean, R. (2009). Photovoice as a Teaching Tool. Learning through with Visual Methods. International Journal of Teaching and Learning in Higher Education, 21, 3, 340-352. https://doi.org/10.1177\%2F2373379917715652

Wahab, A. (2020). Online and Remote Learning in Higher Education Institutes: A Necessity in Light of COVID-19 Pandemic. Higher Education Studies, 10, 3, 16-25. https://doi.org/10.5539/hes.v10n3p16

Wass, R., Anderson, V., Rabello, R., Golding, C., Rangi, A., \& Eteuati, E. (2019). Photovoice as a research method for higher education research, Higher Education Research \& Development, 39,4, 834-850. https://doi.org/10.1080/07294360.2019.1692791

Woodward, S. (2019). Material Methods. London: Sage Publications.

World Economic Forum, (2018). The Future of Jobs Report 2018. http://www3.weforum.org/docs/WEF_Future_of_Jobs_2018.pdf 